
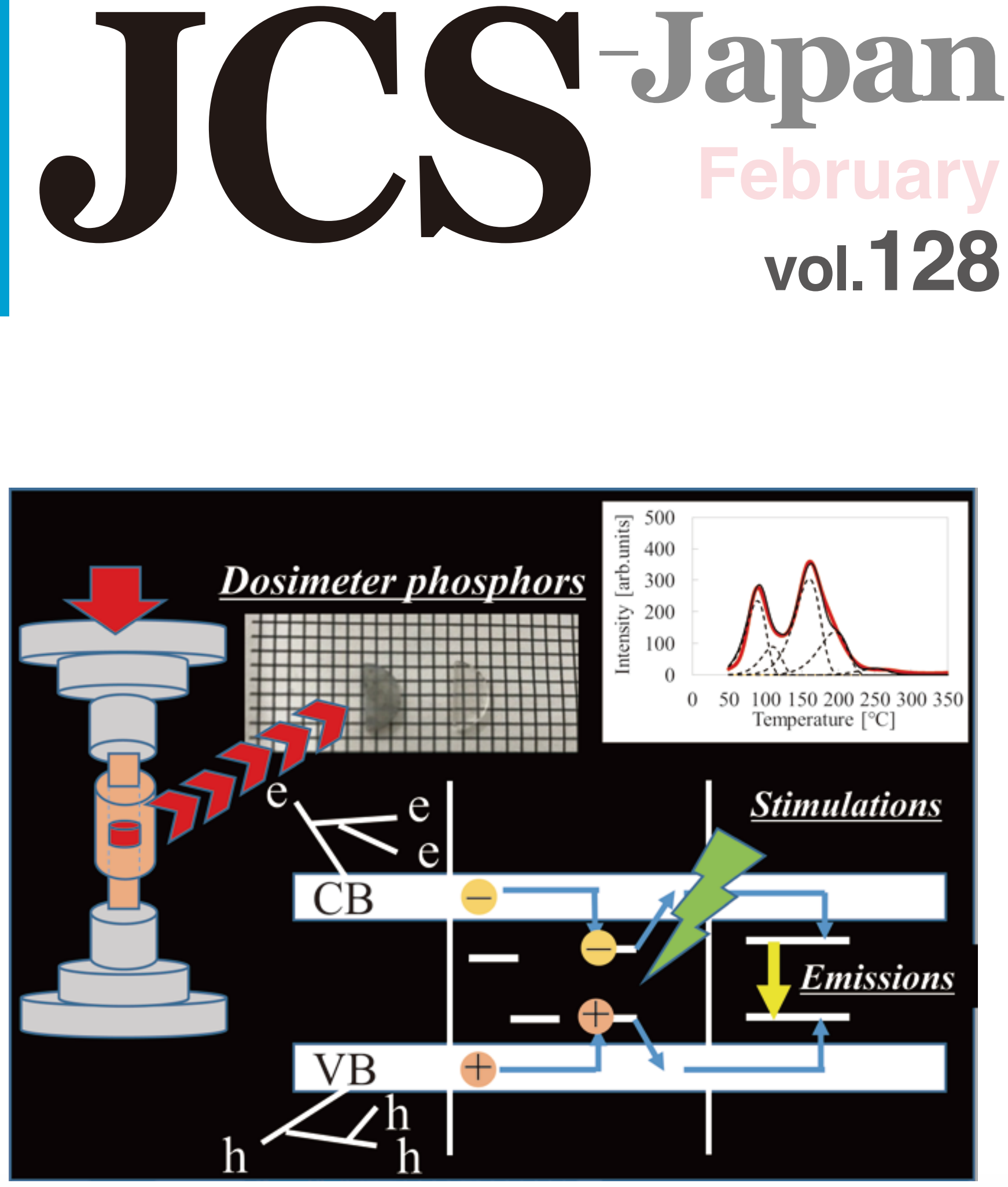

\title{
Journal of the Ceramic Society of Japan
}




\title{
Scintillation and dosimetric properties of Dy-doped $\mathrm{CaF}_{2}$ translucent ceramic and single crystal
}

\author{
Naoki KAWANO ${ }^{1, \dagger}$, Daisuke NAKAUCHI ${ }^{2}$, Fumiya NAKAMURA ${ }^{2}$ and Takayuki YANAGIDA ${ }^{2}$ \\ ${ }^{1}$ Graduate School of Engineering Science, Akita University, Akita 010-8502, Japan \\ ${ }^{2}$ Graduate School of Science and Technology, Nara Institute of Science and Technology (NAIST), Ikoma, Nara 630-0192, Japan
}

\begin{abstract}
We synthesized a Dy-doped $\mathrm{CaF}_{2}$ translucent ceramic by the spark plasma sintering method, and investigated its scintillation and dosimetric properties compared with those of the single crystal. Both the samples showed sharp peaks across $480-670 \mathrm{~nm}$ due to the $4 \mathrm{f}-4 \mathrm{f}$ transitions of $D \mathrm{y}^{3+}$, and a broad peak approximately at $300 \mathrm{~nm}$ due to the self-trapped excitons. The decay time constants of both the samples in scintillation were approximately $5 \mathrm{~ms}$, which was close to those for the $4 \mathrm{f}-4 \mathrm{f}$ transitions of $\mathrm{Dy}{ }^{3+}$. Regarding thermally-stimulated luminescence, the translucent ceramic exhibited higher sensitivity than the single crystal, and the dynamic range was at least 0.01-100 mGy. In addition, both the samples showed optically stimulated luminescence due to the $4 \mathrm{f}-4 \mathrm{f}$ transitions of $\mathrm{Dy}{ }^{3+}$ during $590 \mathrm{~nm}$ stimulation light, but the lowest detection limit of both the samples was approximately $10 \mathrm{mGy}$. (c)2020 The Ceramic Society of Japan. All rights reserved.
\end{abstract}

Key-words : $\mathrm{CaF}_{2}$, Translucent ceramic, Dy, SPS

[Received July 18, 2019; Accepted December 2, 2019]

\section{Introduction}

Dosimeters based on solid-state phosphor materials have received much attention for investigation of accumulated radiation dose, energy and spatial distribution. ${ }^{1)-3)}$ The dosimeters are used in various application fields such as environmental dosimetry, individual radiation monitoring and retrospective accidental dosimetry. ${ }^{1)}$ The phosphor materials for the dosimeters have a capability to store radiation energy as a form of trapping electrons and holes. The absorbed energy can be read out as photon emissions by external stimulation, which decapture the electrons and holes followed by recombination. When stimulated by heat or light, the resulting light is called thermally stimulated luminescence (TSL) or optically stimulated luminescence (OSL), respectively. ${ }^{3)-5)}$ Basic requirements for the phosphor materials include high sensitivity, dose linearity and low fading. To date, many kinds of the phosphor materials such as $\mathrm{Mg}$ and $\mathrm{Ti}$ doped $\mathrm{LiF}, \mathrm{C}$-doped $\mathrm{Al}_{2} \mathrm{O}_{3}$ are used for dosimetric applications. ${ }^{1), 3)}$

$\mathrm{CaF}_{2}$ with an impurity is one of the well-known phosphor materials for radiation dosimetric method owing to high sensitivity and low effective atomic number. For example, Mn-doped $\mathrm{CaF}_{2}$ is commercially known as TLD$400 .{ }^{6)}$ Its TSL sensitivity is much higher than that of TLD100 , and the dynamic range is $0.1 \mu \mathrm{Gy}-100 \mathrm{~Gy}$. It is used for environmental radiation dosimetry and high dose measurement.6),7) Furthermore, Tm-doped $\mathrm{CaF}_{2}$ shows two TSL glow peaks approximately at 150 and $250^{\circ} \mathrm{C}$, and are

Corresponding author: N. Kawano; E-mail: n-kawano@ gipc.akita-u.ac.jp of interest for dose measurements in mixed radiation field since the relative TSL intensity of the glow peaks depends on the linear energy transfer of incident radiation. ${ }^{6)-8)}$ In addition, Dy-doped $\mathrm{CaF}_{2}$ is commercially available as TLD-200. It shows excellent TSL sensitivity, and a linear TSL response over the dose range of $0.1 \mu \mathrm{Gy}-10 \mathrm{~Gy}$. Therefore, it has been use in the field of environmental radiation dosimetry. ${ }^{6), 7)}$ From these examples, the phosphor materials based on $\mathrm{CaF}_{2}$ with an impurity can be one of the promising materials for dosimeter materials. Up to now, there are many studies on $\mathrm{CaF}_{2}$ with an impurity in various forms such as crystals and ceramics, ${ }^{6)}$ but a few studies in a form of translucent ceramics, although the translucent ceramics have some advantages such as high luminescence intensity, high uniformity and high mechanical strength. ${ }^{9)-11)}$

In this study, we synthesized a Dy-doped $\mathrm{CaF}_{2}$ translucent ceramic by the spark plasma sintering (SPS) method, and investigated scintillation and dosimetric properties compared with those of the single crystal. The translucent ceramic synthesized by the SPS could show better dosimetric properties than the single crystal because a large amount of anion vacancies involved in TSL and OSL could be generated in the translucent ceramic due to the synthesis in reducing atmosphere. ${ }^{10)-14)}$ After the synthesis of the translucent ceramic and single crystal, we evaluated their scintillation, TSL and OSL properties.

\section{Experimental methods}

A Dy-doped $\mathrm{CaF}_{2}$ translucent ceramic was synthesized by the SPS method using Sinter Land LabX-100. $\mathrm{CaF}_{2}$ $(99.99 \%)$ and $\mathrm{DyF}_{3}(99.9 \%)$ were mixed in a weight ratio 
of 99.9:0.1. Subsequently, the mixture was loaded in a graphite die and held between graphite punches. In the sintering process, the temperature was increased from 25 to $1070^{\circ} \mathrm{C}$ at a rate of $23^{\circ} \mathrm{C} / \mathrm{min}$ and held for $15 \mathrm{~min}$ under pressure of $70 \mathrm{MPa}$. After that, the surfaces of the ceramic were polished mechanically. Furthermore, a Dy-doped single crystal was synthesized as follows. The mixture of $\mathrm{CaF}_{2}$ and $\mathrm{DyF}_{3}$ in a weight ratio of 99.9:0.1 were put into a carbon crucible, which was set in the SUS chamber of the original furnace (Toei Scientific Industrial). After setting of crucibles and hot zone made of high purity carbon, the chamber was evacuated up to $10^{-4} \mathrm{~Pa}$. Then, the crucible was heated at $400^{\circ} \mathrm{C}$ for 20 min for baking, and the chamber was filled with Ar gas up to ambient pressure. Then, the crucible was heated at the temperature no less than the melting point of $\mathrm{CaF}_{2}$ for $30 \mathrm{~min}$ and cooled naturally. After the mechanical polish, the Dy-doped single crystal was obtained.

Scintillation spectrum under X-ray irradiation was measured using our original setup. ${ }^{10)}$ The X-ray generator with a tungsten anode target $(\mathrm{XRB} 80 \mathrm{P} \& \mathrm{~N} 200 \times 4550$, Spellman) was operated by applying a voltage of $80 \mathrm{kV}$ and a tube current of $1.2 \mathrm{~mA}$ for X-ray irradiation. The scintillation light from the samples was guided to a spectrometer (DU-420-BU2 CCD and 163 monochromator, Andor) using an optical fiber. Further, scintillation decay time profile was measured using an afterglow characterization system with a pulse X-ray tube. ${ }^{15)}$ The time resolution was a few ns, and the detection wavelength range of the photomultiplier tube (R7400P-06, Hamamatsu photonics) was across $160-650 \mathrm{~nm}$. As a dosimetric property, TSL glow curve was measured using a spectrometer (TL2000, Nanogray). ${ }^{16)}$ The temperature range was from 50 to $350^{\circ} \mathrm{C}$, and the heating rate was $1^{\circ} \mathrm{C} / \mathrm{s}$. In addition, OSL spectra and OSL decay time profile were measured using a spectrometer (FP-8600, JASCO). The stimulation wavelength was $630 \mathrm{~nm}$, and the monitoring wavelength for the OSL profile was $480 \mathrm{~nm}$.

\section{Results and discussion}

Figure 1 represents the photograph of the Dy-doped translucent ceramic and single crystal. The thickness of both the samples was about $1.0 \mathrm{~mm}$. The single crystal

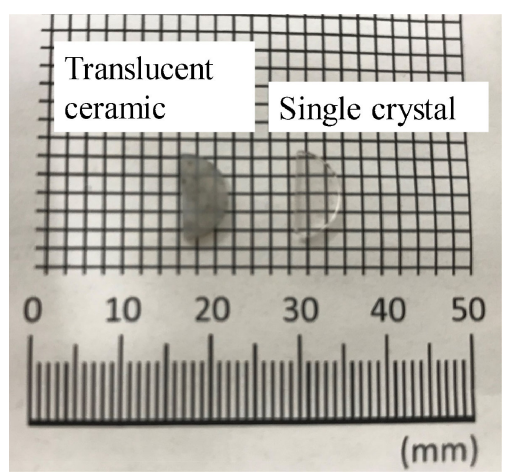

Fig. 1. Photograph of the Dy-doped translucent ceramic and single crystal. seemed to be highly transparent, and have no cracks and defects such as grain boundaries. However, the transparency of the translucent ceramic was not so high that the check pattern on the back of the translucent ceramic was not clearly observed, whereas the pattern beneath the single crystal could be seen through.

Figure 2 shows the in-line transmittance spectra of the Dy-doped translucent ceramic and the single crystal. The single crystal showed high transmittance over $40 \%$ in the spectral range of $350-2700 \mathrm{~nm}$, whereas the translucent ceramic exhibited low transmittance. In addition, no clear absorption peak due to the transitions of Dy ions was observed in both the samples.

Figure 3 exhibits the scintillation spectra of the Dydoped translucent ceramic and single crystal under X-ray irradiation. Sharp emission peaks approximately at 480 $490,570-590,650-670 \mathrm{~nm}$ were observed while a broad peak approximately at $300 \mathrm{~nm}$ was observed in both the samples. ${ }^{17)-20)}$ The spectral features of the sharp emission peaks were similar to those for other $\mathrm{Dy}^{3+}$-doped materials, thus the sharp emission peaks should be ascribed to the $4 \mathrm{f}-4 \mathrm{f}$ transitions of $\mathrm{Dy}^{3+} .{ }^{17)-20)}$ Furthermore, the spectral feature of the broad peak was typical for the selftrapped excitons (STE) in $\mathrm{CaF}_{2}$ host, thus the broad peak was attributed to the STE. ${ }^{12), 21), 22)}$ The scintillation intensity of $480-670 \mathrm{~nm}$ emissions for the translucent ceramic was slightly higher than those for the single crystal. This might be because $\mathrm{Dy}^{3+}$ ions were more uniformly dis-

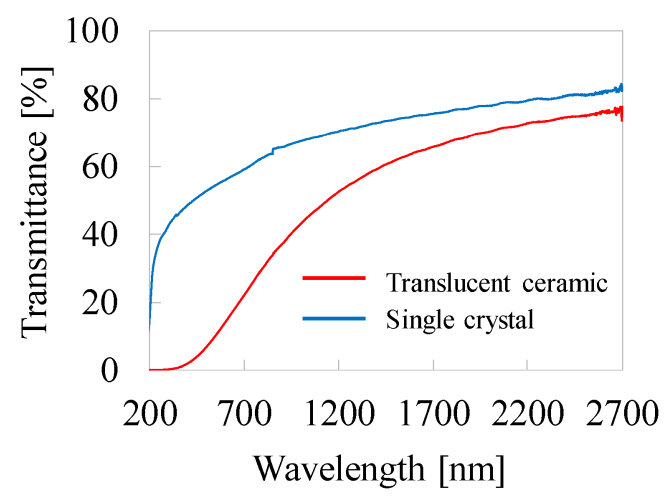

Fig. 2. In-line transmittance spectra of the Dy-doped translucent ceramic and single crystal.

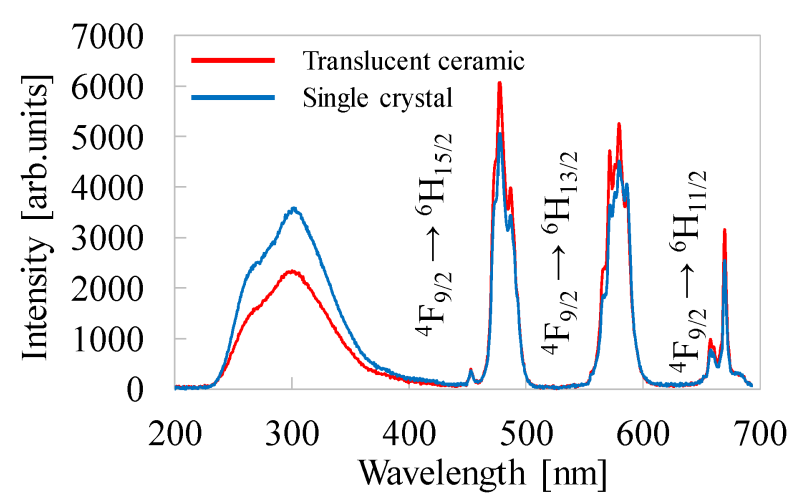

Fig. 3. X-ray induced spectra of the Dy-doped translucent ceramic and single crystal. 

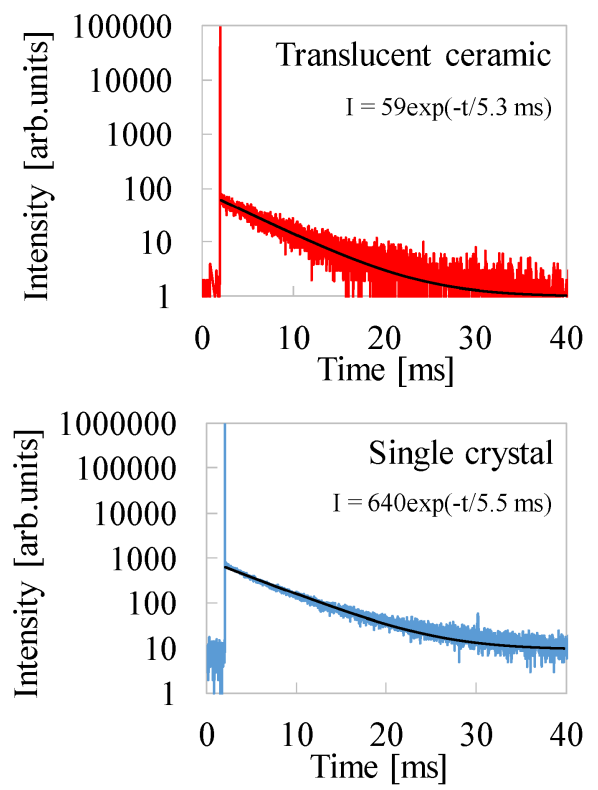

Fig. 4. X-ray induced scintillation decay time profiles of the Dy-doped translucent ceramic and single crystal.

persed in the translucent ceramic compared with the single crystal due to solid state reaction by the SPS method. ${ }^{10}$ )

Figure 4 represents the scintillation decay time profiles of the Dy-doped translucent ceramic and single crystal under X-ray irradiation. Each decay time profile was approximated by an exponential decay function. The derived decay time constants were $5.3 \mathrm{~ms}$ for the translucent ceramic and $5.5 \mathrm{~ms}$ for the single crystal. They were close to the typical decay time constants $(0.1-3.5 \mathrm{~ms})$ for $\mathrm{Dy}^{3+}$-doped $\mathrm{CaF}_{2}$ at $77 \mathrm{~K}$ and the $\mathrm{Dy}^{3+}$-doped materials in photoluminescence (PL). ${ }^{23)-25)}$ Therefore, the emissions should be ascribed to the $4 \mathrm{f}-4 \mathrm{f}$ transitions of $\mathrm{Dy}^{3+}$ since scintillation decay time tends to be longer than PL one due to the additional energy migration processes in scintillation. ${ }^{26}$ Such phenomenon is often observed in various materials. ${ }^{27), 28)}$ In addition, the decay time constants of both the samples in scintillation were almost identical.

Figure 5 shows the TSL glow curves of the Dy-doped translucent ceramic and single crystal. Both the samples were irradiated by X-ray (100 $\mathrm{mGy})$ prior to the measurement. The translucent ceramic exhibited two main glow peaks approximately at 95 and $165^{\circ} \mathrm{C}$ while the single crystal showed several glow peaks over the temperature range of $90-210^{\circ} \mathrm{C}$. Furthermore, the translucent ceramic showed much higher TSL sensitivity than the single crystal. This should be because many defects responsible for TSL were generated in the translucent ceramic during the synthesis of reducing atmosphere. In addition, glow peak temperature, activation energy and frequency factors were calculated by numerical approximation assuming firstorder kinetics as follows. ${ }^{29)}$

$$
\begin{aligned}
I(T)= & I_{\mathrm{M}} \exp \left[1+\frac{E}{k T} \frac{T-T_{\mathrm{M}}}{T_{\mathrm{M}}}-\frac{T^{2}}{T_{\mathrm{M}}^{2}}\left(1-\frac{2 k T}{E}\right)\right. \\
& \left.\times \exp \left(\frac{E}{k T} \frac{T-T_{\mathrm{M}}}{T_{\mathrm{M}}}\right)-\frac{2 k T_{\mathrm{M}}}{E}\right]
\end{aligned}
$$
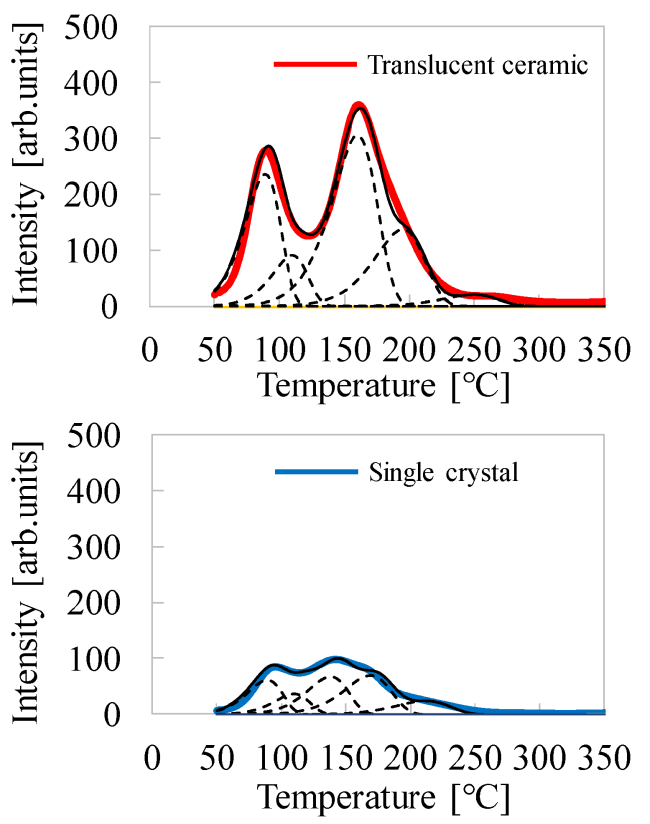

Fig. 5. TSL glow curves of the Dy-doped translucent ceramic and single crystal.

Table 1. Glow peak temperature, activation energy and frequency factors

\begin{tabular}{lcccc}
\hline & & $\begin{array}{c}\text { Glow Peak } \\
\text { Temperature } \\
\left(T_{\mathrm{M}}\right)\left[{ }^{\circ} \mathrm{C}\right]\end{array}$ & $\begin{array}{c}\text { Activation } \\
\text { Energy } \\
(E)[\mathrm{eV}]\end{array}$ & $\begin{array}{c}\text { Frequeucy } \\
\text { Factors } \\
(s)\left[\mathrm{s}^{-1}\right]\end{array}$ \\
\hline \multirow{3}{*}{ Translucent } & Peak1 & 90 & 0.8 & $9.7 \times 10^{9}$ \\
Ceramic & Peak3 & 110 & 0.9 & $5.0 \times 10^{10}$ \\
& Peak4 & 160 & 0.9 & $1.6 \times 10^{9}$ \\
& Peak5 & 250 & 1.0 & $9.0 \times 10^{8}$ \\
& Peak1 & 90 & 0.8 & $9.7 \times 10^{9}$ \\
Single & Peak2 & 110 & 0.9 & $5.0 \times 10^{10}$ \\
Crystal & Peak3 & 140 & 0.9 & $6.7 \times 10^{9}$ \\
& Peak4 & 170 & 0.9 & $9.8 \times 10^{8}$ \\
& Peak5 & 210 & 1.0 & $3.5 \times 10^{8}$ \\
\hline
\end{tabular}

Here, $I(T)$ is the glow peak intensity, $I_{\mathrm{M}}$ is the glow peak top intensity, $T$ is the absolute temperature, $T_{\mathrm{M}}$ is the glow peak temperature, $E$ is the activation energy and $k$ is Boltzmann constant. In addition, the frequency factor can be expressed as $s=\beta E /\left[k\left(T_{\mathrm{M}}\right)^{2}\right] \times \exp \left[E /\left(k T_{\mathrm{M}}\right)\right]$, and the heating rate $\beta$ is $1^{\circ} \mathrm{C} / \mathrm{s} .^{29)}$ The calculation results are summarized in Table 1. Compared with the above two samples and TLD-200 (Dy-doped $\mathrm{CaF}_{2}$ ) in the past study, ${ }^{30)}$ the glow peak temperature and the activation energy were found to be different for each of the three samples. It is suggested that different defects involved in TSL might be generated in each of the three samples. Moreover, the high values of the frequency factors in Table 1 suggested that the thermal ionization should be main detrapping processes for TSL in the translucent ceramic and the single crystal.

Figure 6 shows the TSL dose response curves of the Dy-doped translucent ceramic and single crystal. Here, the integrated intensity of the TSL glow peaks in the temperature range of $50-300^{\circ} \mathrm{C}$ was used as a TSL intensity. Both 


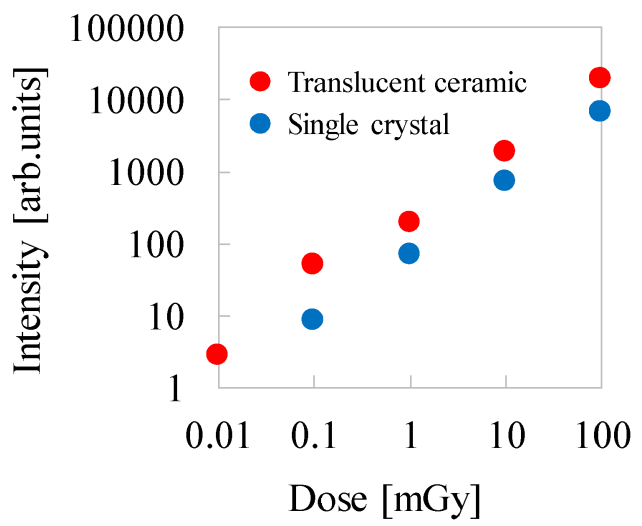

Fig. 6. TSL dose response curves of the Dy-doped translucent ceramic and single crystal.

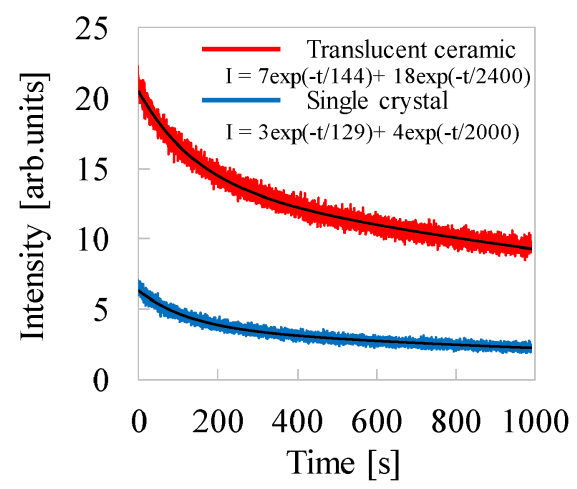

Fig. 7. OSL decay time profiles of the Dy-doped translucent ceramic and single crystal.

the samples showed a linear TSL response against the Xray dose, and the dynamic range of each sample was confirmed to be $0.01-100 \mathrm{mGy}$ for the translucent ceramic and 0.1-100 mGy for the single crystal. The lower detection limit $(0.01 \mathrm{mGy})$ of the translucent ceramic was found to be comparable to that of commercialized TSL dosimeter (TLD-100). ${ }^{6}$ It should be noted that our measurement setup did not allow dose delivery lower than $0.01 \mathrm{mGy}$, and the TSL signals from the translucent ceramic and single crystal were saturated in the TSL reader under X-ray irradiation over $100 \mathrm{mGy}$.

In addition to the TSL properties, we also investigated the OSL properties. Figure 7 represents the OSL decay time profiles of the Dy-doped translucent ceramic and the single crystal after X-ray irradiation of $1 \mathrm{~Gy}$. The stimulation and monitoring wavelengths were 630 and $480 \mathrm{~nm}$, respectively. The signal intensity decreased gradually over time, suggesting that the signals were derived from OSL. In addition, the decay time profiles were approximated by two exponential decay functions. The obtained decay time constants were approximately 144 and $2400 \mathrm{~s}$ for the translucent ceramic, and 129 and $2000 \mathrm{~s}$ for the single crystal. These results suggested that there were at least two detrapping processes involved in OSL in both the samples. Figure 8 shows the OSL spectra of the Dy-doped translucent ceramic and single crystal. Both the samples were irradiated by X-rays (1 Gy) before the measurement.

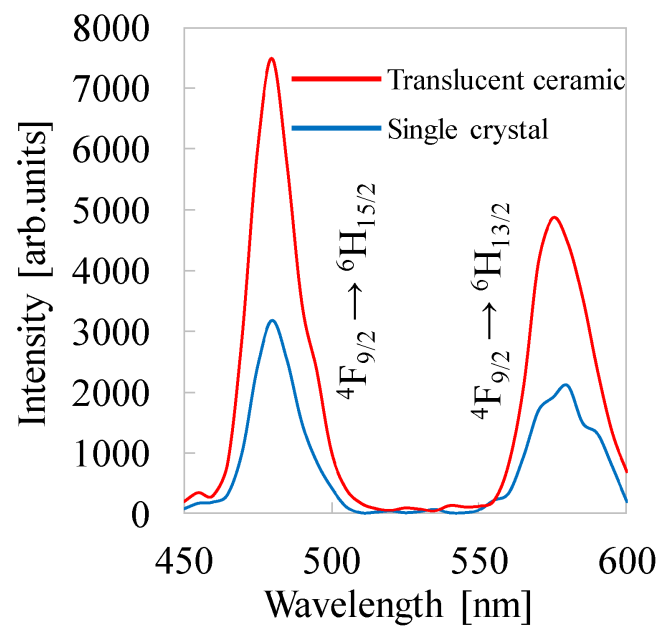

Fig. 8. OSL spectra of the Dy-doped translucent ceramic and single crystal.

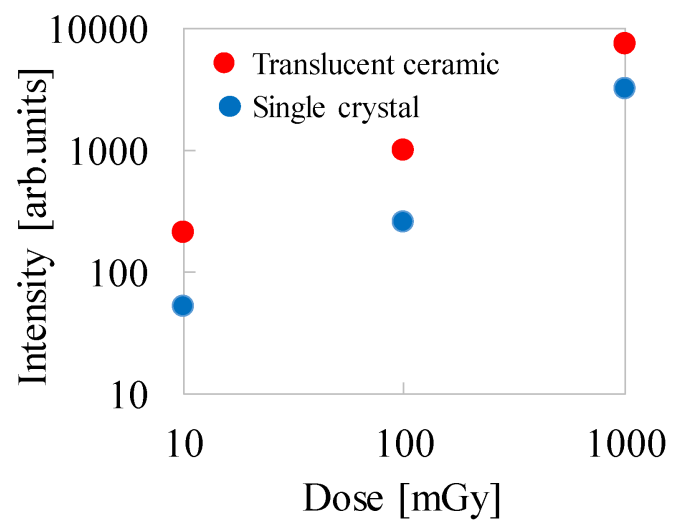

Fig. 9. OSL dose response curves of the Dy-doped translucent ceramic and single crystal.

Emission peaks approximately at 480 and $580 \mathrm{~nm}$ were observed in both the samples during the optical stimulation $(630 \mathrm{~nm})$. The spectral features were similar to those for the $\mathrm{Dy}^{3+}$ doped materials in PL, ${ }^{17)-20)}$ thus the OSL was attributed to the $4 \mathrm{f}-4 \mathrm{f}$ transitions of $\mathrm{Dy}^{3+}$. In addition, the OSL intensity of the translucent ceramic was much higher than that of the single crystal as well as the TSL results shown in Fig. 5. Figure 9 shows the OSL dose response curves of the Dy-doped translucent ceramic and the single crystal. The peak top intensity was defined as the OSL intensity. A linear correlation between X-ray dose and the OSL intensity was observed in both the samples. However, the lowest detection limit of both the samples was about $10 \mathrm{mGy}$, which was much higher than that of conventional OSL materials such as C-doped $\mathrm{Al}_{2} \mathrm{O}_{3}$. ${ }^{1)}$ Throughout this study, we evaluated scintillation and dosimetric properties of the Dy-doped translucent ceramic and single crystal, and the dosimetric properties of the translucent ceramic were superior to those of the single crystal. Furthermore, the translucent ceramic showed excellent TSL sensitivity comparable to commercial TSL dosimeter (TLD-100), thus the Dy-doped $\mathrm{CaF}_{2}$ translucent ceramic should be promising candidate for TSL materials. 


\section{Conclusion}

We characterized scintillation, TSL and OSL properties of the Dy-doped $\mathrm{CaF}_{2}$ translucent ceramic and single crystal. The Dy-doped samples exhibited scintillation due to the STE and the $4 \mathrm{f}-4 \mathrm{f}$ transitions of $\mathrm{Dy}^{3+}$. Further, the Dy-doped translucent ceramic showed higher TSL sensitivity than the single crystal, and its TSL dose range was confirmed to be $0.01-100 \mathrm{mGy}$. In addition, OSL due the $4 \mathrm{f}-4 \mathrm{f}$ transitions of $\mathrm{Dy}^{3+}$ was observed in both the samples, but the OSL sensitivity was much lower than that of common OSL materials.

Acknowledgements This work was supported by Grantin-Aid for Scientific Research (A) (17H01375) and Grantin-Aid for Research Activity Start-up (17H06867) from the Ministry of Education, Culture, Sports, Science and Technology of the Japanese government (MEXT).

\section{References}

1) B. C. Bhatt, Radiat. Prot. Environ., 34, 6-16 (2011).

2) M. R. Mayhugh, R. W. Christy and N. M. Johnson, J. Appl. Phys., 41, 2968-2976 (1970).

3) S. W. S. McKeever, Radiat. Meas., 46, 1336-1341 (2011).

4) A. J. J. Bos, Nucl. Instrum. Meth. B, 184, 3-28 (2001).

5) T. Yanagida, J. Lumin., 169, 544-548 (2016).

6) Data sheet of TLD materials from thermoscientific, https://assets.thermofisher.com/TFS-Assets/LSG/Catalogs/ Dosimetry-Materials-Brochure.pdf.

7) V. E. Kafadar, A. N. Yazaci and R. G. Yildirim, Nucl. Instrum. Meth. B, 267, 3337-3346 (2009).

8) G. Massillon-JL, I. Gamboa-deBuen, A. E. Buenfil, M. A. Monroy-Rodriguez and M. E. Brandan, Nucl. Instrum. Meth. B, 266, 772-780 (2008).

9) S. F. Wang, J. Zhang, D. W. Luo, F. Gu, D. Y. Tang, Z. L. Dong, G. E. B. Tan, W. X. Que, T. S. Zhang, S. Li and L. B. Kong, Prog. Solid State Ch., 41, 20-54 (2013).

10) T. Yanagida, K. Kamada, Y. Fujimoto, H. Yagi and T. Yanagitani, Opt. Mater., 35, 2480-2485 (2013).

11) N. Kawano, T. Kato, G. Okada, N. Kawaguchi and T. Yanagida, Opt. Mater., 73, 364-370 (2017).
12) N. Kawano, D. Nakauchi, F. Fukuda, G. Okada, N. Kawaguchi and T. Yanagida, Jpn. J. Appl. Phys., 57, 102401 (2018).

13) T. Kato, N. Kawano, G. Okada, N. Kawaguchi, K. Fukuda and T. Yanagida, Optik, 168, 956-962 (2018).

14) N. Kawano, T. Kato, G. Okada, N. Kawaguchi and T. Yanagida, Opt. Mater., 88, 67-73 (2019).

15) T. Yanagida, Y. Fujimoto, T. Ito, K. Uchiyama and K. Mori, Appl. Phys. Express, 7, 062401 (2014).

16) T. Yanagida, Y. Fujimoto, N. Kawaguchi and S. Yanagida, J. Ceram. Soc. Jpn., 121, 988-991 (2013).

17) R. Sharma and A. S. Rao, J. Non-Cryst. Solids, 495, 85-94 (2018).

18) S. S. Babu, P. Babu, C. K. Jayasankar, Th. Troster, W. Sievers and G. Wortmann, Opt. Mater., 31, 624-631 (2009).

19) M. Ayvacikli, A. Ege and N. Can, Opt. Mater, 34, 138142 (2011).

20) A. A. El-Maaref, K. H. S. Shaaban, M. Abdelawwad and Y. B. Saddeek, Opt. Mater., 72, 169-176 (2017).

21) F. Nakamura, T. Kato, G. Okada, N. Kawaguchi, K. Fukuda and T. Yanagida, Ceram. Int., 43, 604-609 (2017).

22) F. Nakamura, T. Kato, G. Okada, N. Kawaguchi, K. Fukuda and T. Yanagida, J. Eur. Ceram. Soc., 37, 17071711 (2017).

23) C. T. Kwan, Electronic Theses and Dissertations. 6102 (1971).

24) P. Babu, K. H. Jang, E. S. Kim, L. Shi, R. Vijava, V. Lavin, C. K. Jayasankar and H. J. Seo, J. Non-Cryst. Solids, 356, 236-243 (2010).

25) G. Lakshminarayana, H. Yang, Y. Teng and J. Qiu, J. Lumin., 129, 59-68 (2009).

26) A. Lempicki, A. J. Wojtowicz and E. Berman, Nucl. Instrum. Meth. A, 333, 304-311 (1993).

27) N. Kawano, M. Koshimizu, G. Okada, Y. Fujimoto, N. Kawaguchi, T. Yanagida and K. Asai, Sci. Rep., 7, 14754 (2017).

28) N. Kawano, N. Kawaguchi, K. Fukuda, G. Okada and T. Yanagida, Opt. Mater., 82, 60-64 (2018).

29) G. Kitis, J. M. Gomez-Ros and J. W. N. Tuyn, J. Phys. D Appl. Phys., 31, 2636-2641 (1998).

30) A. N. Yazici, R. Chen, S. Solak and Z. Yegingil, J. Phys. D Appl. Phys., 35, 2526-2535 (2002). 